\title{
Unusual roles of caspase-8 in triple-negative breast cancer cell line MDA-MB-231
}

\author{
ANNA DE BLASIO ${ }^{1}$, RICCARDO DI FIORE ${ }^{1}$, MARCO MORREALE ${ }^{1}$, DANIELA CARLISI ${ }^{2}$, \\ ROSA DRAGO-FERRANTE ${ }^{1}$, MAURO MONTALBANO ${ }^{2}$, CHRISTIAN SCERRI $^{3}$, \\ GIOVANNI TESORIERE ${ }^{4}$ and RENZA VENTO ${ }^{1,4}$ \\ ${ }^{1}$ Laboratory of Biochemistry, Department of Biological, Chemical and Pharmaceutical Sciences and Technologies, \\ ${ }^{2}$ Laboratory of Biochemistry, Department of Experimental Biomedicine and Clinical Neurosciences, \\ University of Palermo, Polyclinic, Palermo, Italy; ${ }^{3}$ Department of Physiology and Biochemistry, \\ University of Malta, Msida, Malta; ${ }^{4}$ Sbarro Institute for Cancer Research and Molecular \\ Medicine, Center for Biotechnology, Temple University, Philadelphia, PA, USA
}

Received November 11, 2015; Accepted December 7, 2015

DOI: 10.3892/ijo.2016.3474

\begin{abstract}
Triple-negative breast cancer (TNBC) is a clinically aggressive form of breast cancer that is unresponsive to endocrine agents or trastuzumab. TNBC accounts for $\sim 10-20 \%$ of all breast cancer cases and represents the form with the poorest prognosis. Patients with TNBC are at higher risk of early recurrence, mainly in the lungs, brain and soft tissue, therefore, there is an urgent need for new therapies. The present study was carried out in MDA-MB-231 cells, where we assessed the role of caspase- 8 (casp-8), a critical effector of death receptors, also involved in non-apoptotic functions. Analysis of casp- 8 mRNA and protein levels indicated that they were up-regulated with respect to the normal human mammalian epithelial cells. We demonstrated that silencing of casp- 8 by small interfering-RNA, strongly decreased MDA-MB-231 cell growth by delaying G0/G1- to S-phase transition and increasing p21, p27 and hypo-phosphorylated/ active form of pRb levels. Surprisingly, casp-8-knockdown, also potently increased both the migratory and metastatic capacity of MDA-MB-231 cells, as shown by both wound healing and Matrigel assay, and by the expression of a number of related-genes and/or proteins such as VEGFA, C-MYC, CTNNB1, HMGA2, CXCR4, KLF4, VERSICAN V1 and MMP2. Among these, KLF4, a transcriptional factor with a dual role (activator and repressor), seemed to play critical roles. We suggest that in MDA-MB-231 cells, the endogenous
\end{abstract}

Correspondence to: Professor Renza Vento, Department of Biological, Chemical and Pharmaceutical Sciences and Technologies, University of Palermo, Polyclinic, Via Del Vespro 129, I-90127 Palermo, Italy

E-mail: renza.vento@unipa.it

Key words: caspase- 8 unusual roles, triple-negative breast cancer cells, MDA-MB-231 cells, cell cycle regulators, caspase-8 knockdown, KLF4, invasivity and metastasis expression of casp-8 might keep the cells perpetually cycling through downregulation of KLF4, the subsequent lowering of p21 and p27, and the inactivation by hyperphosphorylation of $\mathrm{pRb}$. Simultaneously, by lowering the expression of some migratory and invasive genes, casp- 8 might restrain the metastatic ability of the cells. Overall, our findings showed that, in MDA-MB-231 cells, casp-8 might play some unusual roles which should be better explored, in order to understand whether it might be identified as a molecular therapeutic target.

\section{Introduction}

A prominent role of apoptosis is the suicidal destruction of dangerous cells, such as cancer cells. Caspase- 8 (casp-8) is an initiator caspase, initially identified as a critical effector of death receptors, present in many normal and cancerous cell types (1). However, casp-8 is not always involved in cell death signaling and, in some cancer cells, its activity seems to work under non-apoptotic conditions (2). Since cancer cell growth also depends on evasion of apoptosis, one could argue that, in cancer cells, caspases are frequently inactivated. Conversely, in cancer cells, the executioner and initiator caspases are only rarely mutated in specific types of cancer. In tumors such as colon carcinoma, which progresses through multiple stages, the loss of casp- 8 is rare. Whereas, in tumors at rapid progression such as neuroblastoma $(3,4)$, medulloblastoma (5) and glioblastoma (6), as well as in small cell lung carcinoma (7), lack of casp-8 expression is very frequent. Inactivation of the casp- 8 gene was also reported for human hepatocellular carcinomas (8) and advanced gastric cancer (9).

Breast cancer (BC) is the second leading cause of cancer death in women worldwide. It is exceeded only by lung cancer. In year 2015, the estimated number of new cases of invasive breast cancer expected in the United States is 231,840, and $\sim 40,000$ women are expected to die from breast cancer in the same year (10). Triple-negative breast cancer (TNBC), that accounts for $\sim 10-20 \%$ of all breast cancer cases (11), is clini- 
cally aggressive form of $\mathrm{BC}$, which do not express estrogen, progesterone and HER-2/neu receptors (12); as a consequence, they are unresponsive to endocrine agents or trastuzumab (13) and are treated with combined therapies such as surgery, radiation therapy and chemotherapy (14). Overall, TNBC represents the poorest prognosis form and patients with TNBC are at higher risk of early recurrence, mainly in the lungs, brain, and soft tissue (15). Thus, there is an urgent need for new therapies.

Recently, we focused our studies on TNBC cells where the effects of various anticancer drugs were evaluated, showing the potent cytotoxic effect exerted by parthenolide and by SAHA-TRAIL and SAHA-parthenolide combination, and also elucidating their molecular mechanism of action (16-19). Here, we aimed at determining the role played by casp- 8 in TNBC MDA-MB-231 cells. We evaluated mRNA and protein levels of casp- 8 showing that, with respect to the normal human breast epithelial cell line HMEC, there was an overexpression of casp-8. To further evaluate the role of casp-8 in MDA-MB-231 cells, we silenced casp- 8 using a specific small interferingRNA (si-RNA). Under these conditions, we demonstrated that casp-8-knockdown decreased MDA-MB-231 cell growth rate and delayed G0/G1 to S-phase transition; moreover, it potently increased the expression of the cell cycle inhibitors p21 and p27 and those of the hypophosphorylated-active form of pRb. It also augmented the migratory and invasive capacity of the cells and the expression of a number of related genes. Altogether, our results showed that, in MDA-MB-231 cells, casp-8 played some non-canonical roles which should be better explored to understand whether it might be identified as a molecular therapeutic target.

\section{Materials and methods}

Cell cultures. The human breast cancer cell line MDA-MB-231 was purchased from ICLC (Interlab Cell Line Collection, Genova, Italy). Cells were maintained in DMEM/high glucose, supplemented with $10 \%$ heat-inactivated fetal bovine serum (FBS), L-glutamine (2 mM), non-essential amino acid solution $(1 \%)$, penicillin $(100 \mathrm{IU} / \mathrm{ml})$ and streptomycin $(100 \mu \mathrm{g} / \mathrm{ml})$ (Euroclone, Pero, Italy). HMEC (human mammary epithelial cells) were purchased from Lonza (Lonza, Euroclone) and maintained according to the manufacturer's instructions. Cells were incubated in a humidified atmosphere of $95 \%$ air plus $5 \% \mathrm{CO}_{2}$ at $37^{\circ} \mathrm{C}$. Media were replaced every three days. Cellular morphology was evaluated using a Leica DM IRB inverted microscope (Leica Microsystems S.r.l., Milan, Italy); photographs were captured with a computer-imaging system (Leica DC300F camera).

Pharmacological inhibition of caspases. MDA-MB-231 cells were seeded in 6-well plates with a density of $1 \times 10^{5} /$ well in DMEM as described above. After $24 \mathrm{~h}$ from seeding, cells were treated with the pan-caspases inhibitor z-VAD-FMK $(50 \mu \mathrm{M})$ (Promega, Milan, Italy) or the specific caspase- 8 inhibitor z-IETD-FMK (50 $\mu \mathrm{M})$ (Santa Cruz Biotechnology, Santa Cruz, CA, USA) and incubated for $24 \mathrm{~h}$.

Growth curve and cell viability assays. Total cell number and viability were evaluated by trypan blue (Sigma-Aldrich) exclusion counting as previously described (20).
EdU cell proliferation assay. EdU staining was conducted using Click-iT ${ }^{\mathrm{TM}}$ EdU Alexa Fluor imaging kit (Invitrogen, Life Technologies Ltd., Monza, Italy) according to the manufacturer's protocol. Briefly, cells were seeded at density of $5 \times 10^{3}$ cells/well in 96-well plates and, after silencing procedures, cells were incubated with $10 \mu \mathrm{M}$ EdU solution for $2 \mathrm{~h}$. Then, cells were washed in PBS, fixed in $3.7 \%$ formaldehyde in PBS for $15 \mathrm{~min}$ at RT. After washing with $3 \%$ bovine serum albumin (BSA) in PBS, cells were permeabilized with $0.5 \%$ Triton X-100 in PBS for 20 min. Permeabilization buffer was removed washing cells with $3 \%$ BSA in PBS and then incubated with Click-it reaction cocktail prepared as indicated in instruction's kit, for 30 min protecting from light. The cells were washed with $3 \%$ BSA in PBS. Then, cells were counterstained with $5 \mu \mathrm{g} / \mathrm{ml}$ Hoechst 33342 for 30 min for DNA staining. The slides were washed three times with PBS and examined under a fluorescent microscope Leica DM-IRB.

Transient downregulation of casp- 8 by small interfering RNA (siRNA). Cells were seeded into 6-well plates with a density of $1 \times 10^{5}$ well in DMEM-high glucose as previously described. Transfection procedure was performed $24 \mathrm{~h}$ after seeding; specific siRNAs directed against casp-8 (Santa Cruz Biotechnology) were transfected for $5 \mathrm{~h}$ into the cells at a final concentration of $50 \mathrm{nM}$, in the presence of $5 \mu$ l Lipofectamine 2000 suspension (Invitrogen, Life Technologies Ltd.) in a final volume of $1 \mathrm{ml}$ antibiotic- and serum-free DMEM. At the end, the reaction was stopped replacing the culture medium with DMEM + 10\% FBS. Cells were examined for casp- 8 downregulation and other properties, at the time indicated in the single experiments. siRNA, consisting in a scramble sequence, was used as a negative control.

Western blot analysis. Cells were detached by trypsinization (0.025\% trypsin-EDTA in PBS) and lysed in RIPA buffer containing protease inhibitors cocktail (Sigma-Aldrich S.r.l., Milan, Italy) for $30 \mathrm{~min}$ and sonicated three times for $10 \mathrm{sec}$. Protein concentration was examined by Bradford Protein Assay (Bio-Rad Laboratories S.r.l., Segrate, Milan, Italy). Then, $20 \mu \mathrm{g}$ of total proteins were resolved by SDS-PAGE and transferred to a nitrocellulose membrane (Bio-Rad). Immunodetection analysis was performed using primary antibodies against caspase-8 (\#9746), p21 (\#2946), p27 (\#2552) by Cell Signaling Technology, Euroclone. pRb (sc-102), VEGFA (sc-7269), HMGA2 (sc-30223), KLF4 (sc-20691), CXCR4 (sc-53534), MMP2 (sc-10736) by Santa Cruz Biotechnology. Versican V1 (ab19345) by Abcam Inc. Prodotti Gianni, Milan, Italy) and the appropriate horseradish peroxidase-conjugated secondary antibodies (Santa Cruz). Immunoreactive signals were detected using enhanced chemiluminescence (ECL) reagents (Bio-Rad). The correct protein loading was confirmed by stripping the immunoblot and reprobing with primary antibody for actin (SigmaAldrich). Bands were visualized and photographed with Chemi Doc XRS (Bio-Rad). Quantification was performed using Quantity One software.

Flow cytometry analysis. Cell cycle and proliferation analyses were performed as previously described (21). 


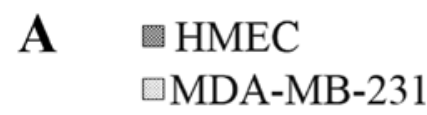

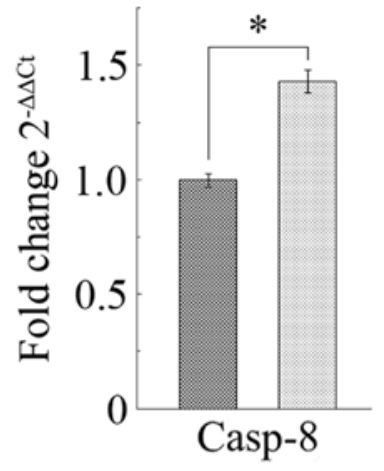

\section{B $\square$ Control $\square \mathrm{z}-\mathrm{VAD}-\mathrm{FMK}$ -Z-IETD-FMK}

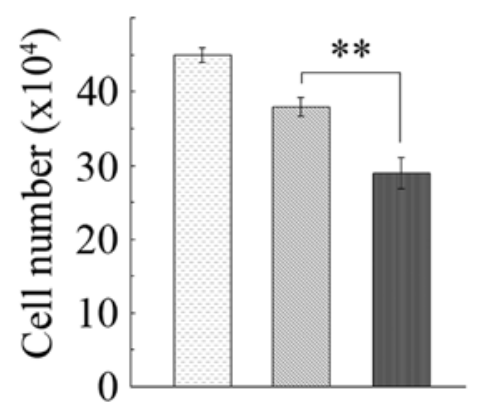

RNA extraction and real-time RT-PCR. RNA was extracted with RNeasy mini kit and reverse transcription reaction was done using QuantiTect Reverse Transcription kit (Qiagen, Milan, Italy), according to the manufacturer's instructions. Briefly, $0.8 \mu \mathrm{g}$ RNA was reverse transcribed incubating a $20-\mu 1$ reaction mix in a thermal cycler at $42^{\circ} \mathrm{C}$ for $25 \mathrm{~min}$. The cDNA was used for quantitative analysis by real-time PCR using iQ-SYBR Green Supermix (BioRad) and PCR primers (QuantiTect Primer assay; Qiagen). The primers used were: casp-8 (QT00052416), P21 (QT00062090), P27 (QT00998445), RB1 (QT00066899), VEGFA (QT01010184), cMYC (QT00035406), CTNNB1 (QT00077882), KLF4 (QT00061033), HMGA2 (QT01157674), CXCR4 (QT00223188). Thermal cycling protocol was programmed as follows: $95^{\circ} \mathrm{C}$ for $10 \mathrm{~min} ; 95^{\circ} \mathrm{C}$ for $30 \mathrm{sec}$, $60^{\circ} \mathrm{C}$ for $60 \mathrm{sec}, 72^{\circ} \mathrm{C}$ for $30 \mathrm{sec}$ for 40 cycles and a final extension at $72^{\circ} \mathrm{C}$ for $5 \mathrm{~min}$. To determine primer specificity, three stages $\left(95^{\circ} \mathrm{C}\right.$ for $15 \mathrm{sec}, 60^{\circ} \mathrm{C}$ for $20 \mathrm{sec}$ and $95^{\circ} \mathrm{C}$ for $15 \mathrm{sec}$, with a ramping time of $20 \mathrm{~min}$ ) were added at the end of the PCR to obtain dissociation curves for each gene. Melting curve analyses were done for all samples. No RT control was included to verify RNA signal specificity, for each gene tested. All real-time PCR reactions are performed in triplicate. Real-time PCR and data collection were performed on an IQ5 cycler instrument (Bio-Rad) qPCR data were analyzed by IQ5 cycler software. The relative amount of mRNAs was normalized to GAPDH (QT01192646). Data were calculated using the comparative $2^{-\Delta \Delta C t}$ method.

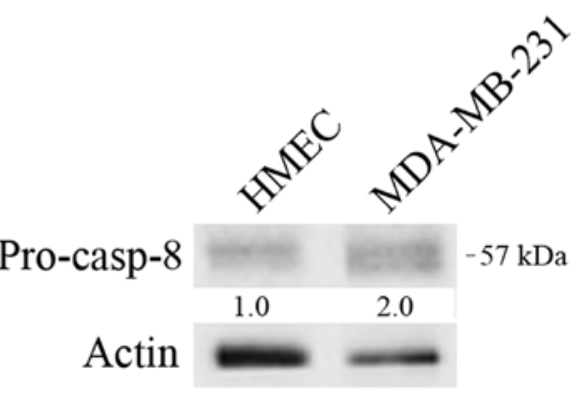

Figure 1. Evaluation of caspase-8 expression in HMEC and MDA-MB-231 cells. (A) Real-time RT-PCR (left panel) and western blot (right panel) analyses of casp-8 in HMEC and MDA-MB-231 cells. Data represent the mean with standard deviation $(\mathrm{n}=4) ;{ }^{*} \mathrm{P}<0.05$ as compared with HMEC cells analysis of Casp-8 in HMEC and MDA-MB-231 cells. Protein expression level relative to HMEC cells is shown below the blot. The results are representative of four independent experiments. (B) Analysis of total cell number in MDA-MB-231 cells after 24-h treatment with $50 \mu \mathrm{M} \mathrm{z}$-VAD-FMK or $50 \mu \mathrm{M}$ z-IETD-FMK. Data represent the mean with standard deviation $(\mathrm{n}=4) ;{ }^{* *} \mathrm{P}<0.01$ as compared with control cells.
Wound healing assay. For the wound healing assay, MDA-MB-231 cells were transfected with caspase-8-siRNA or scramble siRNA. After $24 \mathrm{~h}$, transfected cells were harvested, counted and re-seeded into 6-well plates at the density of $10^{6}$ cells/well. After $24 \mathrm{~h}$, the confluent cells were scratched with a 200- $\mu 1$ pipette tip to generate the wound. One hour before scratching, the medium was replaced with medium containing $0.1 \%$ FBS to minimize the cell proliferation. Cells were monitored for $24 \mathrm{~h}$ post-wounding using Leica DM-IRB microscope; images were acquired on a Leica DC300F digital camera using Leica IM50 software. The wound area closure was determined by Image J software and reported as percentage of the total area of wound as previously described (20).

Transwell migration assay. For the Transwell migration assay, MDA-MB-231 cells were transfected with casp-8-siRNA or scrsiRNA. After $24 \mathrm{~h}$, transfected cells were harvested, counted and re-seeded at $1 \times 10^{5}$ cells/well in the upper chamber of a 6-well invasion chamber system (BD Biosciences, Discovery Labware, Becton-Dickinson, Buccinasco, Italy), in serum-free DMEM. DMEM supplemented with 10\% FBS was placed in the bottom well as chemoattractant. Plates were incubated at $37^{\circ} \mathrm{C}$ with $5 \% \mathrm{CO}_{2}$, in humidified air for $48 \mathrm{~h}$. Non-migratory cells in the upper chamber were then removed with a cottontip applicator. Migrated cells on the lower surface were stained with Hoechst 33342 (2.5 $\mu \mathrm{g} / \mathrm{ml}$; Sigma-Aldrich) for $10 \mathrm{~min}$ and then visualized under a fluorescence microscope. The number 
A

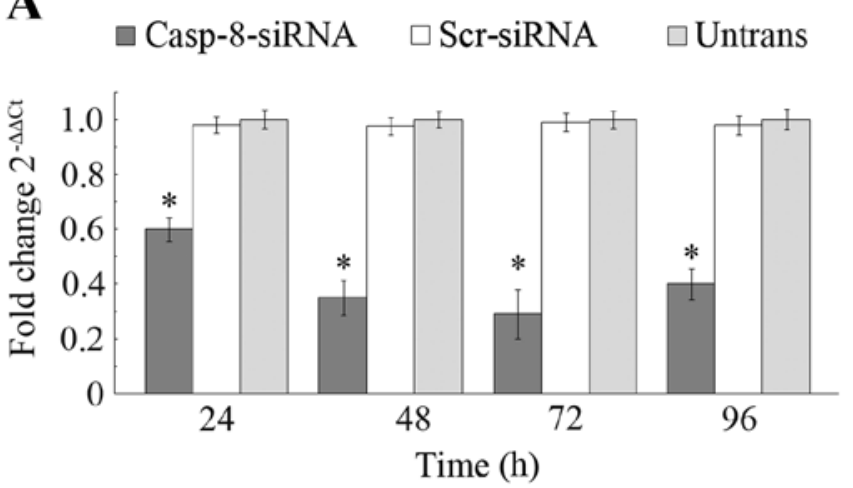

C

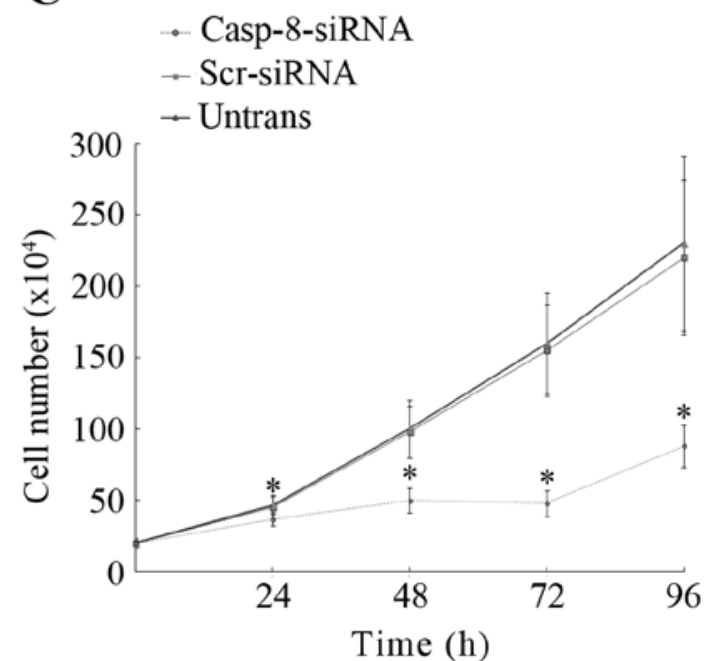

B

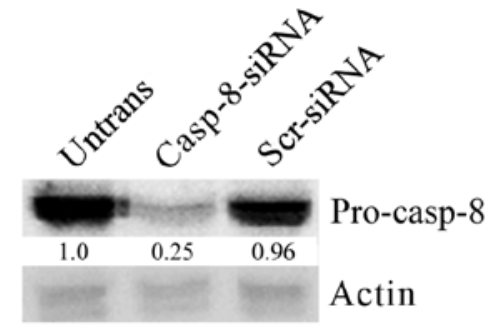

D

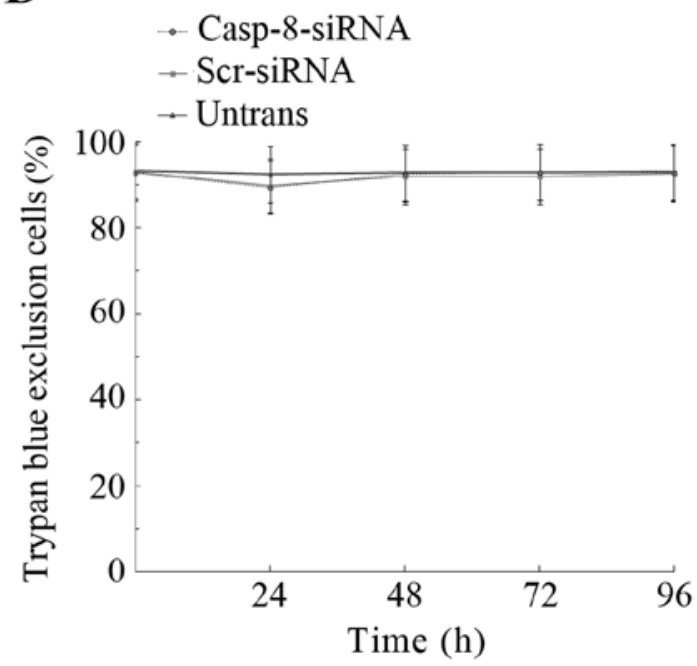

Figure 2. Evaluation of knockdown efficiency of caspase-8 and its effect on MDA-MB-231 cell proliferation. (A) Cells transfected with casp-8-siRNA or scr-siRNA, were compared with untransfected cells. Expression of casp- 8 was examined by real-time RT-PCR analysis between $24-96$ h; ${ }^{P}<0.05$ as compared with untransfected/scramble-siRNA cells. (B) Western blot analysis of Casp-8 at $72 \mathrm{~h}$ after transfection. Protein expression level relative to untransfected cells is shown below the blot. The results are representative of four independent experiments. (C) Cellular growth and (D) viability of transfected and untransfected cells were evaluated by Trypan blue exclusion method. Data represent the mean with standard deviation $(n=4)$; ${ }^{*}<0.05$ as compared with untransfected cells.

of migrating cells was determined by counting in five random fields on each membrane.

Statistical analysis. Data, presented as mean $\pm \mathrm{SD}$, were analyzed using the two-tailed Student's t-test using Microsoft Excel. Differences were considered significant at $\mathrm{P}<0.05$.

\section{Results}

Expression of casp-8 in MDA-MB-231 breast cancer cells compared with normal human breast epithelial cell line HMEC. We initially evaluated the levels of casp-8 in MDA-MB-231 breast cancer cells in comparison with nontransformed human mammary epithelial cells HMEC. As Fig. 1A shows, casp-8 mRNA was expressed in both cell lines, however, in MDA-MB-231 cells, the levels were much higher (1.4-fold) than in HMEC cells. The figure also shows (western blot analysis) that the levels of casp- 8 protein were significantly higher in MDA-MB-231 than in HMEC cells. As a preliminary molecular approach to study the involvement of casp- 8 in the apoptotic cascade, we evaluated the effects of Z-VAD-FMK, an irreversible pan-caspase inhibitor, and of
Z-IETD-FMK, a specific casp-8 inhibitor, on MDA-MB-231 cell number. Surprisingly, we observed (Fig. 1 B) that the treatment determined a decrease in cell number, whereas it did not induce loss of cell viability (data not shown). In particular, after 24-h treatment, Z-VAD-Fmk determined a decrease in cell number of $\sim 15 \%$, while Z-IETD-FMK determined a decrease of $\sim 35 \%$. As a results of the inhibitory effects on canonical caspase, in contrast, an increase in cell number was expected, while the observed decreases suggested that, in TNBC MDA-MB-231 cells, casp- 8 could exert unusual roles. To assess such hypothesis, we silenced casp-8.

Casp-8-knockdown by casp-8-siRNA. As reported in Materials and methods, casp- 8 was transiently depleted by small interfering RNA (si-RNA). After transfection of the cells with casp-8-siRNA or scrambled siRNA (scr-siRNA), the level of endogenous casp-8 mRNA was checked. Between 24 and $96 \mathrm{~h}$ after casp-8 siRNA transfection, qRT-PCR (Fig. 2A) shows that, in cells transfected with casp-8-siRNA, the levels of casp- 8 mRNA progressively lowered with respect to both untransfected cells and cells transfected with scr-siRNA. The effect of gene silencing was already evident 
$24 \mathrm{~h}$ after transfection (40\% decrease of casp- 8 mRNA), increased after $48 \mathrm{~h}(65 \%)$ and reached its maximum after $72 \mathrm{~h}$, when a $71 \%$ decrease was observed. Thereafter, casp- 8 mRNA level increased, so that at $96 \mathrm{~h}$ after transfection, its decreased value was $\sim 60 \%$. This suggested that, at that time, the transient silencing started to recover and that the optimal silencing efficiency was maintained until $72 \mathrm{~h}$ after transfection. Western blot analysis, which was performed at $72 \mathrm{~h}$ after transfection (Fig. 2B), showed that casp- 8 protein decreased by $75 \%$, suggesting that it was reduced similarly to its mRNA. Importantly, the knockdown of mRNA and protein was specific as no reduction was observed in cells transfected with scr-siRNA. Thus, $72 \mathrm{~h}$ of casp-8-knockdown was the silencing time employed for our subsequent experiments.

Casp-8-knockdown strongly reduced MDA-MB-231 growth rate without affecting cell viability. To evaluate whether casp-8-knockdown modified the growth rate of MDA-MB-231 cells, untransfected cells and cells transfected with scr-siRNA or with casp-8 siRNA were analysed (0-96 h) for cell proliferation. Through cell counting, we discovered (Fig. 2C) that upon casp-8 knockdown, cell number progressively lowered with respect to untransfected or scr-siRNA-transfected cells. In particular, at 24, 48 and $72 \mathrm{~h}$ after transfection, growth rate decreased by 20,50 and $70 \%$ respectively; then, at $96 \mathrm{~h}$, the decrease slightly diminished (62\%) with an overall trend, which strongly agreed with what was observed for casp- 8 mRNA after the casp-8 si-RNA transfection. Interestingly, the strong reduction in growth rate induced by casp-8-knockdown did not affect cell viability, as the trypan blue exclusion assay showed (Fig. 2D).

Casp-8-knockdown delays G0/G1 to S-phase transition. The evaluation of the effect of casp-8-knockdown on cell cycle, in order to distinguish cells in the different phases of the cell cycle, was performed by flow cytometric analysis of cellular DNA content in casp- 8 silenced cells. The analysis was also performed in both untransfected and scr-siRNAtransfected cells. In Fig. 3A, the DNA histogram displays that casp-8-knockdown induced a significant accumulation of cells in G0-G1 phase (11\%), with corresponding decrease in percentage of S phase and G2-M phases.

For all the results obtained so far, both untransfected and scr-siRNA-transfected samples were used as control. However, due to the fact that statistically significant differences $(\mathrm{P}>0.05)$ have not been observed between untransfected cells and cells transfected with the scr-siRNA, henceforth, only scr-siRNA were used as control. Under these conditions, cells were microscopically observed at $72 \mathrm{~h}$ post casp- 8 transfection and analysed for cells in the S-phase of the cell cycle (EdU positivity). This assay, which allows for a more accurate detection of DNA synthesis in proliferating cells, showed that, in agreement with cell cycle study, casp-8-knockdown cells were strongly less EdU-positive than control cells (Fig. 3B). Overall, these findings indicate that in MDA-MB-231 cells, casp-8 knockdown consistently reduced cell proliferation by delaying G0-G1 to S phase transition.

Casp-8-knockdown increases the expression of key cell cycle regulators. To reveal the molecular mechanism of cell cycle arrest in the G0/G1 phase, the key molecular protagonists of cell cycle control were evaluated, particularly, the level of RB1 gene expression and of p21 and p27, the cyclin-CDK complex inhibitors. As reported in Fig. 3C, quantification by qRT-PCR analysis of RB1, p21 and p27, showed that, in MDA-MB-231 cells, casp-8-knockdown increases their expression by $2.1,3.8$ and 2.4-fold, respectively. Moreover, by western blot analysis, the level of the corresponding proteins was evaluated. As reported in Fig. 3D, the analysis of the protein $\mathrm{pRb}$ allowed us to identify two distinct molecular forms migrating at different electrophoretic speeds, a slower form (upper band) and a faster form (lower band). These two forms might correspond to the hyper-phosphorylated-inactive form ( $\mathrm{ppRb}$ ) and to the hypophosphorylated-active form ( $\mathrm{pRb}$ ), respectively. As the figure shows, casp-8-knockdown increased the hypophosphorylated/ active form of the protein, while it strongly decreased the levels of the hyperphosphorylated/inactive form. The same figure also shows that, the levels of p21 and p27, similarly to their mRNA levels, strongly increased after casp-8-knockdown. Densitometric analysis of the described proteins is reported in Fig. 3E. Overall, these results suggest that the delay in G0-G1 to S-phase transition caused by casp- 8 knockdown might arise from the strong upregulation of p21 and p27, with consequent decrease in $\mathrm{pRb}$ phosphorylation.

Casp-8 knockdown affects metastatic capacity of $M D A-M B-231$ cells. To assess the metastatic capacity of MDA-MB-231 cells, the gene expression profiles of the metastatic markers after casp- 8 knockdown were also measured. As reported in Fig. 4A, the qRT-PCR analysis of a panel of metastatic markers showed a potent increase in the expression of all the metastatic stimulator genes studied. In particular, casp-8 knockdown increased the expression of VEGFA (2.6-fold), C-MYC (2.4-fold), CTNNB1 (1.4-fold), Krüppel-like factor 4 (KLF4) (2.1-fold), HMGA2 (2.9-fold), and CXCR4 (1.7-fold). Western blot analysis of VEGFA, HMGA2, KLF4 and CXCR4, showed similar increases in protein levels (Fig. 4B).

Casp-8 knockdown strongly influences migratory and invasive capacity of $M D A-M B-231$ cells. To evaluate whether casp-8 knockdown influences the motility and invasivness of MDA-MB-231 cells, scratch/wound healing and Matrigel Transwell invasion assays were performed, respectively. In agreement with the increased metastatic properties induced by casp- 8 knockdown, the data from the wound-healing repair assay obtained at 4, 8 and $24 \mathrm{~h}$ after scratching, proved that, in comparison with the control cells, casp- 8 knockdown cells were distinctively more migratory, with a wound closure per field of 1.23-, 1.27- and 1.42-fold $(\mathrm{P}<0.01)$, respectively (Fig. 5A and B). These results were also strengthened by the experiments performed with Matrigel, which showed a significant increase in the invasivity capacity of casp- 8 knockdown cells in comparison with the control cells (Fig. 5C and D).

Recently, it has been shown that versican, a large chondroitin sulfate proteoglycan belonging to the family of lecticans, strongly promotes cell migration and invasion in a number of cancer cells $(22,23)$. Thus, it was pertinent to verify whether such involvement was also possible in MDA-MB-231 cells. Western blot analysis of the levels of versican showed that these cells lightly expressed the versican V1 isoform and 
A

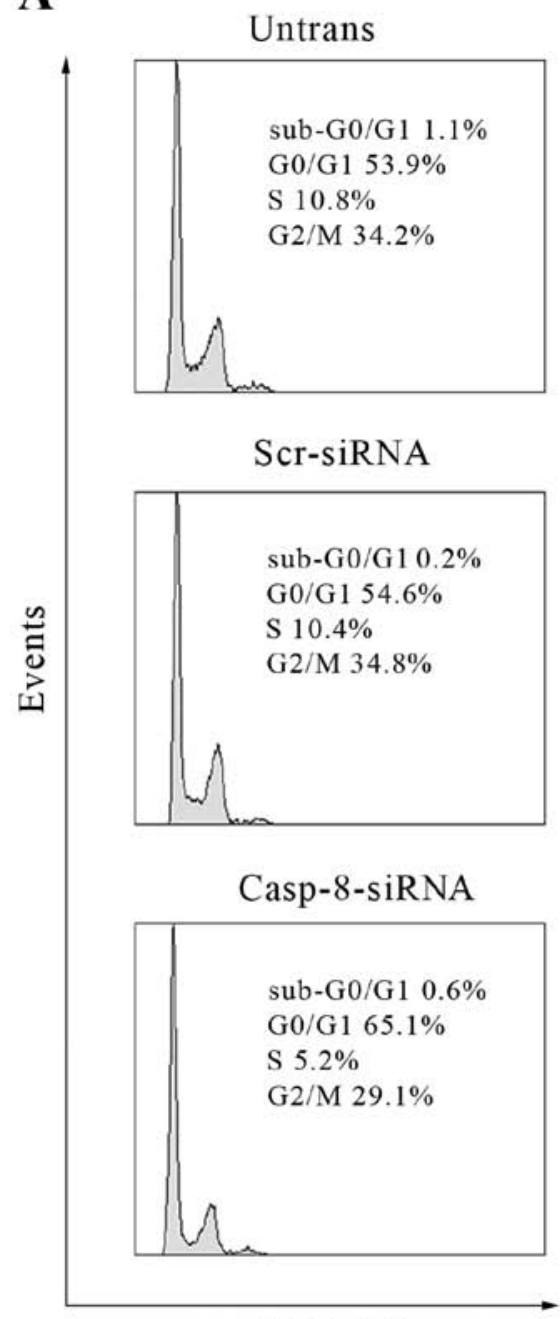

FL3-LIN
B
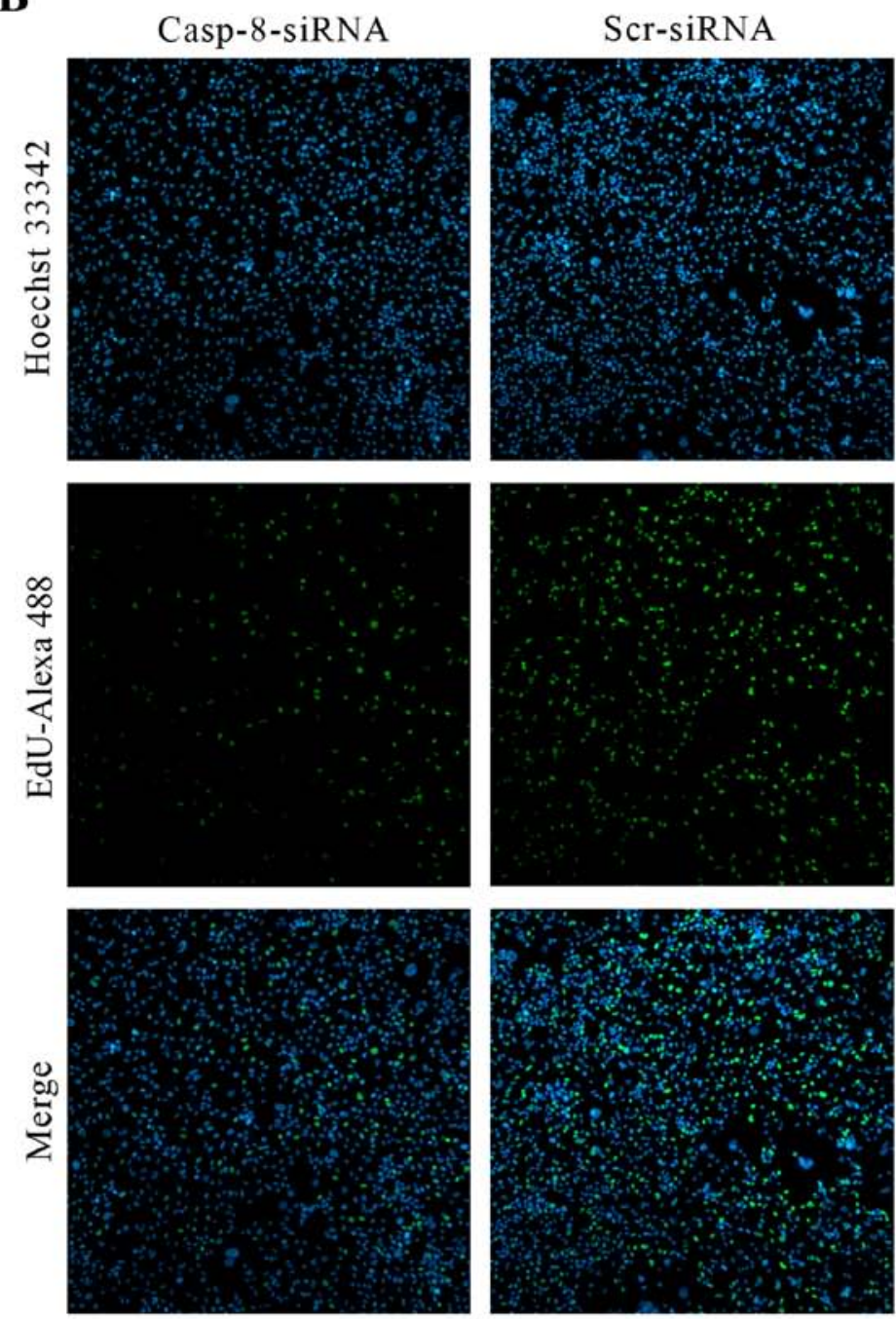

E

$\square \mathrm{pRb}$

$\square \mathrm{ppRb}$
Scr-siRNA

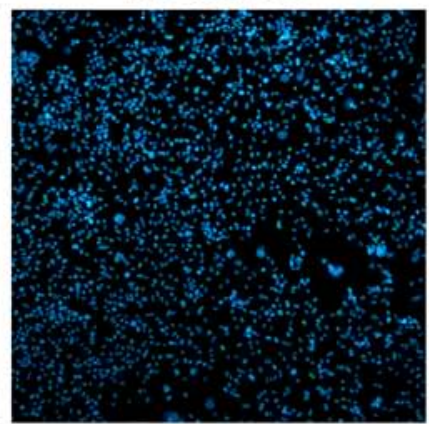

C

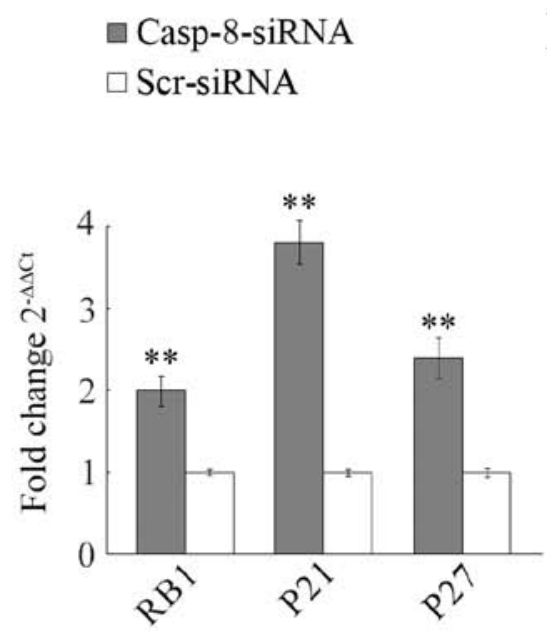

D

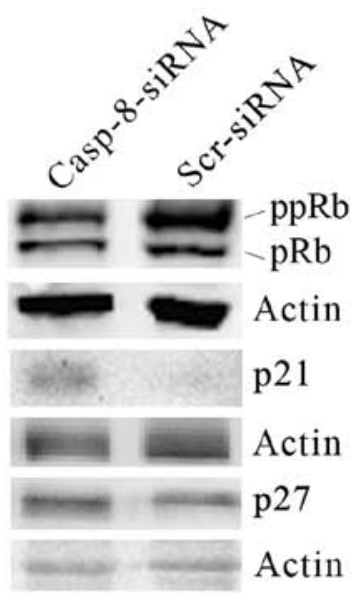


A $\square$ Casp-8-siRNA

$\square$ Scr-siRNA

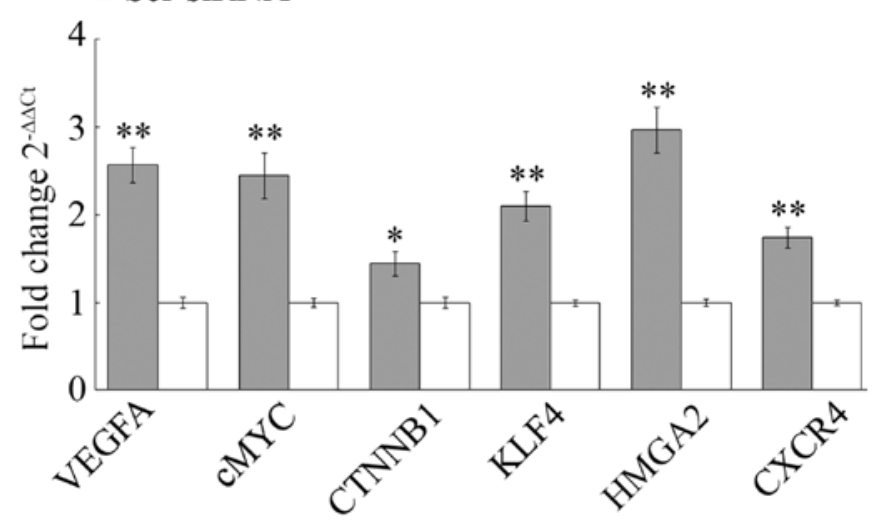

B
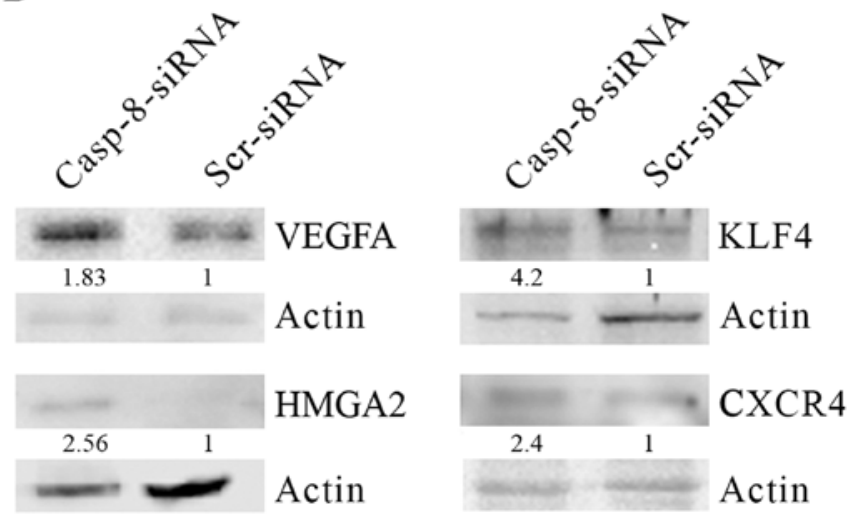

Figure 4. Casp-8-knockdown modulates metastatic properties of MDAMB-231 cells. (A) Real-time RT-PCR analysis of cell migration- and invasion-related markers. Data represent the mean with standard deviation $(\mathrm{n}=4) ;{ }^{*} \mathrm{P}<0.05$ and ${ }^{* *} \mathrm{P}<0.01$ as compared with Scr-siRNA cells. (B) Western blot analysis of some proteins related to the above reported mRNAs. Protein expression levels relative to Scr-siRNA cells are shown below the blot. The results are representative of four independent experiments.

that casp-8 knockdown strongly increased its levels (Fig. 5E). The same figure also demonstrated a strong increase in the levels of the metalloproteinase MMP2. Overall, the data demonstrate that casp-8 knockdown strongly increases migratory and metastatic capacities of MDA-MB-231 cells.

\section{Discussion}

Casp-8 plays an important role as an initiator caspase in apoptosis, therefore, promoting casp-8 expression might seem good for use in cancer treatment. However, assigning to casp-8 a general antitumor role may be misleading, as dysregulation of programmed cell death components could result in a switch to a non-apoptotic signaling pathway to promote tumor growth, survival and cell migration associated with metastasis (2). Moreover, in tumors, casp- 8 is not generally lost and often, its expression is heterogeneous and sometimes very elevated $(24,25)$. Overall, as a number of malignant tumors tolerate very high levels of casp- 8 , it seems likely that the apparent upregulation of this protein in these tumors may represent exploitation of one or more non-apoptotic roles of casp-8 (25). Thus, before any therapeutic strategies are hypothesized to increase casp- 8 expression, the reported knowledge must be taken into account.

In this report, we demonstrated that in MDA-MB-231 breast cancer cell line, casp- 8 exhibits RNA and protein levels markedly higher than in the normal human breast epithelial cell line HMEC.

In the preliminary approach to evaluate the canonical involvement of casp- 8 in apoptosis, we were surprised to find that treatments with casp- 8 inhibitors, rather than causing an increase in cell number, significantly reduced MDA-MB-231 cell proliferation. This addressed our study on non-canonical roles played by casp- 8 in these cells and the results obtained have reconciled this initially unexpected finding by showing that in these cells apoptosis is minimal.

Here, for the first time, we demonstrated that in MDA-MB-231 cells, silencing of casp- 8 by siRNA induced a potent decrease in cell growth rate accompanied by a delayed G0/G1 to S-phase transition. Such a delay was accompanied by a significant increase in the expression of the key cell cycle inhibitors p21 and p27 and by increased levels of the hypophosphorylated-active form of $\mathrm{pRb}$.

It has been reported that during cancer progression, dysregulation of oncogenic or tumor suppressive transcription factors, collectively influenced invasion-metastasis cascade (26). Despite major advances in understanding the molecular and genetic basis of cancer, metastasis remains the cause of $90 \%$ of cancer-related mortality (27). So, understanding the molecular mechanisms of metastasis initiation and progression may be critical for individualizing new therapeutic strategies. Our results showed that the delayed G0/G1-S-phase transition observed in casp-8-knockdown MDA-MB-231 cells, was accompanied by a powerful increase in the expression of a number of factors (VEGFA, C-MYC, CTNNB1, KLF4, HMGA2, CXCR4, VERSICAN V1 and MMP2) known to strongly promote cell migration and metastasis in numerous tumors $(22,23,26,28)$. This could provide MDA-MB-231 cells with particularly high migratory/metastatic capacity, as suggested by wound healing and Transwell assays, showing that, in comparison with control cells, casp-8 knockdown cells were distinctively more migratory, and by the marked increase in the levels of versican $\mathrm{V} 1$, which is known to strongly promote cell migration and invasion in a number of cancer cells. In agreement with this result, we also observed a strong increase in the levels of MMP2, a metalloproteinase, which has been reported to have a close association with tumor size in 135 patients with infiltrative breast carcinomas (28). Among the above-mentioned overexpressed factors, KLF4, known to explicate a dual function, both as a transcriptional activator and repressor, seemed to play pivotal roles in mediating cell growth inhibition. Indeed, KLF4 may bind and activate the promoters of the p21 and p27 thus, blocking cell cycle progression from the G1 phase to $S$ phase (29-32). Interestingly, in 100 patients with breast cancer, where the expression of KLF4 was determined by tissue microarray analysis, it has been shown that KLF4 is a favorable prognostic indicator (33).

In conclusion, in light of the unusual roles played by casp- 8 in various cancer cell types, and of the results by us obtained in MDA-MB-231 cells after casp-8-knockdown, we suggest that in MDA-MB-231 cells, casp- 8 could be sucked towards 
A

Time (h)
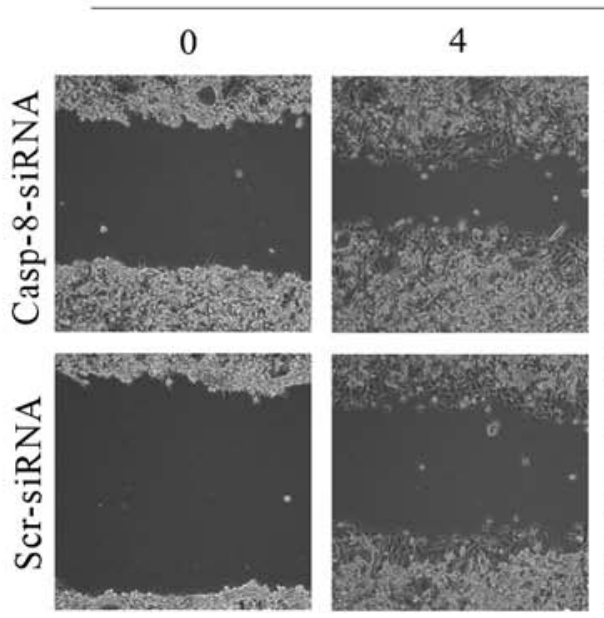

C

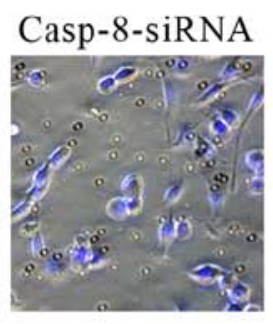

4

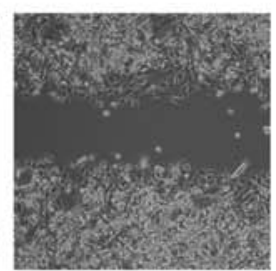

$\mathbf{F}$

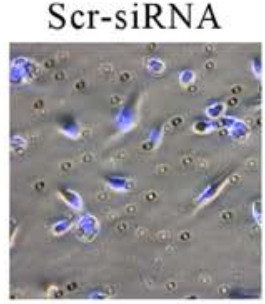

8
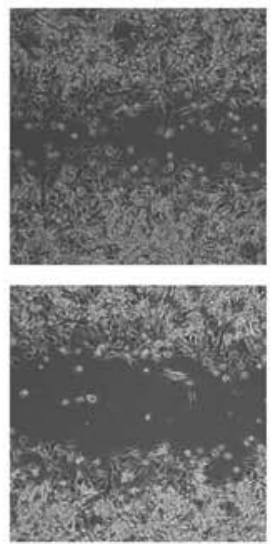

D
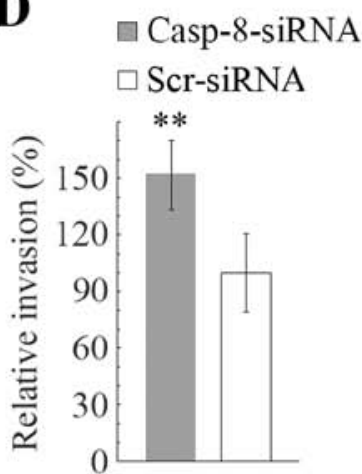

B

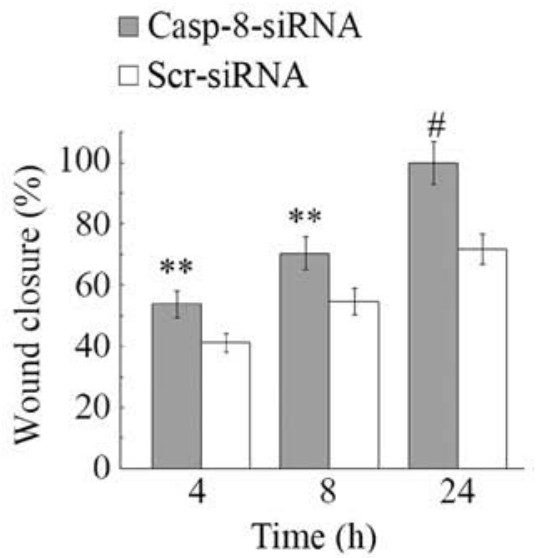

$\mathbf{E}$

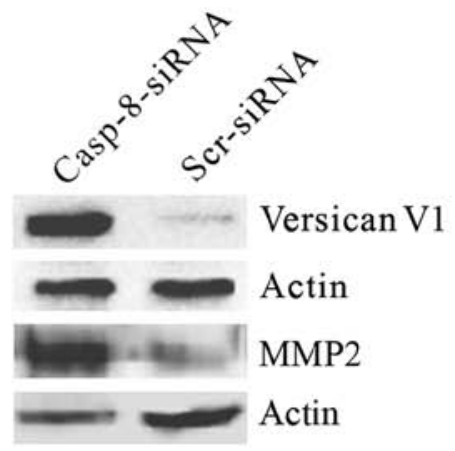

Figure 5. Effects of Casp-8-knockdown on migratory and invasive capacity of MDA-MB-231 cells. (A) Representative phase contrast images from the woundhealing assay (magnification, x100). (B) Quantification of the scratch wound-healing assay. The extent of wound closure was quantified by measuring the wound area in comparison with the initial wound area. Data represent the mean with standard deviation $(\mathrm{n}=4) ;{ }^{* *} \mathrm{P}<0.01$ and ${ }^{\#} \mathrm{P}<0.001$ as compared with scr-siRNA cells. (C) Representative images from the Transwell invasion assay. At $48 \mathrm{~h}$ after incubation, cells migrated to the underside of the insert, were stained with Hoechst 33342 (magnification, x200). (D) Graph summarizing Transwell invasion (referred as average number of casp-8-siRNA cells relative to scr-siRNA cells). Data represent the mean with standard deviation $(n=4) ;{ }^{* *} \mathrm{P}<0.01$ as compared with scr-siRNA cells. (E) Western blot analysis of Versican V1 and MMP2. Images are representative of four independent experiments. (F) Schematic roles of caspase-8 in MDA-MB-231 cells. 
non-canonical functions. In these cells, casp- 8 could be aimed at maintaining a balance between cell proliferation and invasiveness. To this aim, downregulating KLF4, which in turn may downregulate p21 and p27 with consequent hyperphosphorylation and inactivation of $\mathrm{pRb}$, would be an excellent machinery exploited by casp- 8 to keep MDA-MB-231 cells permanently in cycle. Simultaneously, casp-8 could tightly control the levels of the stimulatory aggressiveness and invasiveness as VEGFA, C-MYC, CTNNB1, HMGA2, CXCR4, VERSICAN V1 and MMP2, so that the cancer cells could balance growth rate and invasiveness (Fig. 5F). We believe that the unusual roles played by casp- 8 in MDA-MB-231 cells should be further explored, in order to understand whether and how it might be identified as a molecular therapeutic target.

\section{Acknowledgements}

This study was supported in part by the European Regional Development Fund, European Territorial Cooperation 2007-2013,CCI2007 CB 163 PO037,OPItalia-Malta 2007-2013 and by the Italian Ministry of Education, University and Research (MIUR) ex-60\%, 2012; Dr D. Carlisi was a recipient of a fellowship granted by MIUR (contract no. 82, January 23, 2014); Dr R. Di Fiore and Dr R. Drago-Ferrante were recipients of fellowships granted by the European Regional Development Fund, European Territorial Cooperation 2007-2013, CCI 2007 CB 163 PO 037, OP Italia-Malta 2007-2013; Dr M. Montalbano is a PhD student supported by 'Italian Ministry of Education, University and Research' (MIUR); Dr M. Morreale is a recipient of a contract for research support granted by the European Regional Development Fund, European Territorial Cooperation 2007-2013, CCI 2007 CB 163 PO 037, OP ItaliaMalta 2007-2013.

\section{References}

1. Kim HS, Lee JW, Soung YH, Park WS, Kim SY, Lee JH, Park JY, Cho YG, Kim CJ, Jeong SW, et al: Inactivating mutations of caspase-8 gene in colorectal carcinomas. Gastroenterology 125 : 708-715, 2003.

2. Torres VA, Mielgo A, Barbero S, Hsiao R, Wilkins JA and Stupack DG: Rab5 mediates caspase-8-promoted cell motility and metastasis. Mol Biol Cell 21: 369-376, 2010.

3. Stupack DG, Teitz T, Potter MD, Mikolon D, Houghton PJ, KiddVJ, Lahti JM and Cheresh DA: Potentiation of neuroblastoma metastasis by loss of caspase-8. Nature 439: 95-99, 2006.

4. Teitz T, Wei T, Valentine MB, Vanin EF, Grenet J, Valentine VA, Behm FG, Look AT, Lahti JM and Kidd VJ: Caspase 8 is deleted or silenced preferentially in childhood neuroblastomas with amplification of MYCN. Nat Med 6: 529-535, 2000.

5. Pingoud-Meier C, Lang D, Janss AJ, Rorke LB, Phillips PC, Shalaby $\mathrm{T}$ and Grotzer MA: Loss of caspase- 8 protein expression correlates with unfavorable survival outcome in childhood medulloblastoma. Clin Cancer Res 9: 6401-6409, 2003.

6. Skiriute D, Vaitkiene P, Saferis V, Asmoniene V, Skauminas K, Deltuva VP and Tamasauskas A: MGMT, GATA6, CD81, DR4 and CASP8 gene promoter methylation in glioblastoma. BMC Cancer 12: 218, 2012.

7. Hopkins-Donaldson S, Ziegler A, Kurtz S, Bigosch C, Kandioler D, Ludwig C, Zangemeister-Wittke U and Stahel R: Silencing of death receptor and caspase- 8 expression in small cell lung carcinoma cell lines and tumors by DNA methylation. Cell Death Differ 10: 356-364, 2003.

8. Soung YH, Lee JW, Kim SY, Sung YJ, Park WS, Nam SW, Kim SH, Lee JY, Yoo NJ and Lee SH: Caspase-8 gene is frequently inactivated by the frameshift somatic mutation 1225_1226delTG in hepatocellular carcinomas. Oncogene 24: $141-1 \overline{4} 7,2005$.
9. Soung YH, Lee JW, Kim SY, Jang J, Park YG, Park WS, Nam SW, Lee JY, Yoo NJ and Lee SH: Caspase-8 gene is inactivated by somatic mutations in gastric carcinomas. Cancer Res 65: 815-821, 2005.

10. American Cancer Society: Surveillance and Heath Services Research, 2015. http://www.cancer.org/research/cancerfactsstatistics/cancerfactsfigures2015/

11. Zhang J, Wang Y, Yin Q, Zhang W, Zhang T and Niu Y: An associated classification of triple negative breast cancer: The risk of relapse and the response to chemotherapy. Int J Clin Exp Pathol 6: 1380-1391, 2013.

12. Bauer KR, Brown M, Cress RD, Parise CA and Caggiano V: Descriptive analysis of estrogen receptor (ER)-negative, progesterone receptor (PR)-negative, and HER2-negative invasive breast cancer, the so-called triple-negative phenotype: a population-based study from the California cancer Registry. Cancer 109: 1721-1728, 2007.

13. Bayraktar S and Glück S: Molecularly targeted therapies for metastatic triple-negative breast cancer. Breast Cancer Res Treat 138: 21-35, 2013.

14. Yagata H, Kajiura Y and Yamauchi H: Current strategy for triplenegative breast cancer: Appropriate combination of surgery, radiation, and chemotherapy. Breast Cancer 18: 165-173, 2011.

15. Pogoda K, Niwińska A, Murawska M and Pieńkowski T: Analysis of pattern, time and risk factors influencing recurrence in triplenegative breast cancer patients. Med Oncol 30: 388, 2013.

16. D'Anneo A, Carlisi D, Lauricella M, Puleio R, Martinez R, Di Bella S, Di Marco P, Emanuele S, Di Fiore R, Guercio A, et al: Parthenolide generates reactive oxygen species and autophagy in MDA-MB231 cells. A soluble parthenolide analogue inhibits tumour growth and metastasis in a xenograft model of breast cancer. Cell Death Dis 4: e891, 2013.

17. Carlisi D, Lauricella M, D'Anneo A, Emanuele S, Angileri L, Di Fazio P, Santulli A, Vento R and Tesoriere G: The histone deacetylase inhibitor suberoylanilide hydroxamic acid sensitises human hepatocellular carcinoma cells to TRAIL-induced apoptosis by TRAIL-DISC activation. Eur J Cancer 45: 2425-2438, 2009.

18. Lauricella M, Ciraolo A, Carlisi D, Vento R and Tesoriere G: SAHA/TRAIL combination induces detachment and anoikis of MDA-MB231 and MCF-7 breast cancer cells. Biochimie 94: 287-299, 2012.

19. Carlisi D, Lauricella M, D'Anneo A, Buttitta G, Emanuele S, di Fiore R, Martinez R, Rolfo C, Vento R and Tesoriere G: The synergistic effect of SAHA and parthenolide in MDA-MB231 breast cancer cells. J Cell Physiol 230: 1276-1289, 2015.

20. Di Fiore R, Marcatti M, Drago-Ferrante R, D'Anneo A, Giuliano M, Carlisi D, De Blasio A, Querques F, Pastore L, Tesoriere G, et al: Mutant p53 gain of function can be at the root of dedifferentiation of human osteosarcoma MG63 cells into 3AB-OS cancer stem cells. Bone 60: 198-212, 2014.

21. Di Fiore R, Drago-Ferrante R, Pentimalli F, Di Marzo D, Forte IM, D'Anneo A, Carlisi D, De Blasio A, Giuliano M, Tesoriere G, et al: MicroRNA-29b-1 impairs in vitro cell proliferation, self-renewal and chemoresistance of human osteosarcoma $3 \mathrm{AB}-\mathrm{OS}$ cancer stem cells. Int J Oncol 45: 2013-2023, 2014.

22. Gorter A, Zijlmans HJ, van Gent H, Trimbos JB, Fleuren GJ and Jordanova ES: Versican expression is associated with tumorinfiltrating CD8-positive T cells and infiltration depth in cervical cancer. Mod Pathol 23: 1605-1615, 2010.

23. Ricciardelli C, Sakko AJ, Ween MP, Russell DL and Horsfall DJ: The biological role and regulation of versican levels in cancer. Cancer Metastasis Rev 28: 233-245, 2009.

24. Jäger R and Zwacka RM: The enigmatic roles of caspases in tumor development. Cancers (Basel) 2: 1952-1979, 2010.

25. Stupack DG: Caspase- 8 as a therapeutic target in cancer. Cancer Lett 332: 133-140, 2013.

26. Ell B and Kang Y: Transcriptional control of cancer metastasis. Trends Cell Biol 23: 603-611, 2013.

27. Weigelt B, Peterse JL and van 't Veer LJ: Breast cancer metastasis: Markers and models. Nat Rev Cancer 5: 591-602, 2005.

28. Nakopoulou L, Tsirmpa I, Alexandrou P, Louvrou A, Ampela C, Markaki S and Davaris PS: MMP-2 protein in invasive breast cancer and the impact of MMP-2/TIMP-2 phenotype on overall survival. Breast Cancer Res Treat 77: 145-155, 2003. 
29. Tiwari N, Meyer-Schaller N, Arnold P, Antoniadis H, Pachkov M van Nimwegen E and Christofori G: Klf4 is a transcriptional regulator of genes critical for EMT, including Jnk1 (Mapk8). PLoS One 8: e57329, 2013.

30. Shi Y and Ai W: Function of KLF4 in stem cell biology. In: Biochemistry, Genetics and Molecular Biology 'Pluripotent Stem Cells'. Bhartiya D and Lenka N (eds). InTech, 2013.

31. Rowland BD, Bernards R and Peeper DS: The KLF4 tumour suppressor is a transcriptional repressor of p53 that acts as a context-dependent oncogene. Nat Cell Biol 7: 1074-1082, 2005.
32. Wei D, Kanai M, Jia Z, Le X and Xie K: Kruppel-like factor 4 induces $\mathrm{p} 27 \mathrm{Kip} 1$ expression in and suppresses the growth and metastasis of human pancreatic cancer cells. Cancer Res 68: 4631-4639, 2008.

33. Nagata T, Shimada Y, Sekine S, Hori R, Matsui K, Okumura T, Sawada S, Fukuoka J and Tsukada K: Prognostic significance of NANOG and KLF4 for breast cancer. Breast Cancer 21: 96-101, 2014. 